\title{
Comparison between Mifepristone and Ulipristal acetate as an alternative to surgical management of uterine fibroids (Leiomyoma) in symptomatic patients of reproductive age group in Asian population
}

\author{
Ashish R. Kale* \\ Department of Obstetrics and Gynecology, Ashakiran Hospital and Asha IVF Centre, Pune, Maharashtra, India \\ Received: 23 November 2017 \\ Accepted: 02 December 2017 \\ *Correspondence: \\ Dr. Ashish R. Kale, \\ E-mail: drashishkale1978@yahoo.com \\ Copyright: () the author(s), publisher and licensee Medip Academy. This is an open-access article distributed under \\ the terms of the Creative Commons Attribution Non-Commercial License, which permits unrestricted non-commercial \\ use, distribution, and reproduction in any medium, provided the original work is properly cited.
}

\begin{abstract}
Background: Present study was a randomised prospective observational study carried out at Ashakiran Hospital and IVF centre Pune, Maharashtra, India to evaluate the efficacy, safety, and quality of life by using Ulipristal acetate 10 $\mathrm{mg}$ and Mifepristone $25 \mathrm{mg}$ daily doses to treat uterine fibroids of two subgroups involving fibroids $<3 \mathrm{~cm}$ and fibroids 3 to $5 \mathrm{~cm}$ all are in reproductive age group with symptomatic in nature over 3 months.

Methods: A total number of 40 patients were recruited in the study of which they were divided into two groups according to the size of the fibroid as $<3 \mathrm{~cm}$ and $3-5 \mathrm{~cm}$ as seen on transvaginal as well as transabdominal ultrasound. Further they were randomly assigned to either mifepristone or ulipristal orally with each category having 10 patients each to assess changes in fibroid size, in symptomatic pain reduction, menorrhagia and in quality of life.

Results: The 25-mg dosage of Mifepristone is shown to be a good and effective way of treatment in fibroids less than $3 \mathrm{~cm}$ in achieving $40 \%$ reduction in size and 50\% reduction in menorrhagia as compared to Ulipristal $10 \mathrm{mg}$ which acts better in other subgroup of size $3-5 \mathrm{~cm}$ of fibroids.

Conclusions: Still larger RCTs are needed to study the long-term benefits of these drugs.
\end{abstract}

Keywords: Asian population, Mifepristone, Symptomatic fibroids, Ulipristal acetate

\section{INTRODUCTION}

Uterine fibroids are one of the most common benign uterine tumors seen in females of reproductive age group. ${ }^{1}$ They either may be completely asymptomatic (diagnosed incidentally while doing ultrasound for some other reason) or may present as menorrhagia, lower abdominal or back pain, pelvic mass, obstructive uropathy, anemia secondary to blood loss and infertility. ${ }^{2}$ In some patient these symptoms may severely affect the quality of life. The incidence of uterine fibroids is variable because most of them are asymptomatic hence remain undetected for years. Some studies have suggested that more than $75 \%$ women of age above 50 years may have symptomatic or asymptomatic fibroids.
The percentage of patients having symptomatic uterine fibroids is reported to range from $25-30 \%$. $^{3}$

The exact etiology of fibroids is debatable but many factors are reported to have some role in the pathogenesis of fibroids including genetic, hormonal and biological factors. The risk factors for developing fibroids include obesity, nulliparity, younger age at menarche and African race. $^{4}$

The diagnosis of uterine fibroids is usually done by ultrasound examination which usually shows a welldefined hypoechoic lesion within myometrium having a characteristic peripheral vascularity on Doppler examination. ${ }^{5}$ The sensitivity of transvaginal ultrasound 
is more that transabdominal ultrasound in the diagnosis of small fibroids. ${ }^{6}$ On T1 weighted images (MRI) these uterine fibroids may appear as low to intermediate signal intensity areas as compared to the normal myometrium. On T2 weighted images they appear as low signal intensity lesions. ${ }^{7}$

The complications associated with uterine fibroids may include severe anemia requiring intervention in the form of transfusions, hyaline or red degeneration, urinary retention, hydronephrosis secondary to obstructive uropathy and rarely sarcomatous changes..$^{8,9}$

The treatment of uterine fibroids depends upon the size, symptoms, location and age of the patient. Patients who are asymptomatic and in whom there are very minimal symptoms these fibroids should be left alone, and no active intervention is required. ${ }^{10}$ In patients having severe symptoms affecting quality of life considerable surgical management may be required. Minimally invasive surgeries like hysteroscopic myomectomy (for submucosal fibroids), Laparoscopic myomectomy (for symptomatic subserosal and less commonly for intramural fibroids), abdominal myomectomy and hysterectomy (when woman no longer wishes to preserve uterus or fertility like in perimenopausal women or in women where sarcomatous changes are suspected on imaging). ${ }^{11}$ Other less invasive procedure include uterine artery embolization and magnetic resonance guided focused ultrasound surgery (MRgFUS). ${ }^{12}$

Medical management is used in patients for short term relief and as pre-operative adjunct treatment for reduction of size of the fibroid. Nonetheless many of the studies have come up with the conclusion that medical management may be used for small sized fibroids. Various medical therapies used for fibroids include tranexamic acid, combined oral contraceptive pills, $\mathrm{GnRH}$ analogs, selective estrogen and progesterone receptor modulators, Somatostatin analogs and aromatase inhibitors. ${ }^{13}$ Various selective progesterone receptor modulators (SPRMs) used for uterine fibroids include Mifepristone and Ulipristal Acetate. Many researchers have individually studied the effect of mifepristone and Ulipristal acetate on fibroids and found that both these selective progesterone receptor modulators (SPRMs) are effective for management of fibroids. ${ }^{14}$ Mifepristone has been in use since 2002 while Ulipristal acetate has recently been used for fibroids. ${ }^{15}$ The comparative studies comparing these 2 SPRMs are rare hence we conducted this prospective case control study to compare efficacy of Mifepristone and Ulipristal acetate as an alternative to surgical management of symptomatic uterine fibroids

Uterine Leiomyoma (fibroids) are benign tumors arising from the smooth muscles and connective tissue in the uterus. By reproductive age group more than $60 \%$ females are expected to have symptomatic or asymptomatic uterine fibroids. They may present as menorrhagia, lower abdominal pain, frequency of micturition, pregnancy loss, dysparinuea and backache. Many of the times these fibroids are asymptomatic and may be detected in patients undergoing ultrasound examination for some other reason.

The management of uterine fibroids depends upon factors like possibility of pregnancy in future, whether preservation of uterus is desirable, severity and characteristics of symptoms. Various treatment options include observation and follow up (in small asymptomatic fibroids), medical management (Mifepristone or Ulipristal acetate), uterine fibroid embolisation and hysterectomy. Use of Mifepristone and Ulipristal acetate individually has been studied by some researchers but comparative studies of these 2 drugs have rarely been done. For this reason, we have conducted this study to compare efficacy of Mifepristone and Ulipristal acetate as an alternative to surgical management of symptomatic uterine fibroids.

\section{METHODS}

This was a prospective case control study conducted at a tertiary care hospital situated in an urban area. A total number of 40 patients with symptomatic fibroids were enrolled in this study. The patients were divided into two groups (Group A and B) of 20 patients each according to whether they received either Mifepristone or Ulipristal acetate orally. The primary outcome was evaluated in terms of change in size of fibroid, reduction in pain, resolution of menorrhagia and improvement in quality of life.

The study was approved by institutional ethical committee. The informed consent was obtained from all the patients before enrolling them in the study.

Detailed history and demographic profile was noted in all the cases. Menstrual blood loss was assessed by taking into account number of pads soaked, degree of soakage and passage of clots. Any history of dysmenorrhea, dyspareunia or menorrhagia was noted. A complete general and systemic examination followed by gynecological examination was done.

Routine investigations like complete blood count, renal and hepatic functions tests were done in all patients. Transvaginal ultrasound examination was done to know the exact number and location of fibroids. Patients were divided into 2 groups (Group A and Group B). Group A patient received using mifepristone $25 \mathrm{mg}$ and Group B patients received Ulipristal acetate $10 \mathrm{mg}$. Patients were followed up for 3 months and the parameters like change in fibroid size, reduction in pain, resolution of menorrhagia and improvement in quality of life was compared between these 2 groups.

Results were tabulated and analyzed using SPSS 16.0 version software. Microsoft word and excel were used for generating charts and graphs. 


\section{Inclusion criteria}

- Females diagnosed to be having uterine fibroids in reproductive age group

- Patients having symptoms like menorrhagia, dysmenorrhea, abdominal pain or any other symptoms related to fibroids

- Single fibroid

- Patients having consented to be part of this study.

\section{Exclusion criteria}

- Pregnant females

- Those who refused consent

- Renal or hepatic dysfunction or any other disease in which either mifepristone or Ulipristal is contraindicated

- Patients having adenomyosis, endometrial hyperplasia or genital tract infections

- Not taken any previous medication.

\section{RESULTS}

The administration of both drugs, Mifepristone and Ulipristal acetate, in women with symptomatic fibroids was associated with decreased pain, reduced blood loss and decreased size of fibroids. While it was found that Mifepristone was more effective in patients having smaller fibroids (less than $3 \mathrm{~cm}$ ), Ulipristal acetate was more effective in medical management of the patients having fibroids of relatively larger size $(3-5 \mathrm{~cm})$. Treatment with Ulipristal acetate was associated with significant pain reduction in patients having fibroid size of $3-5 \mathrm{~cm}(60 \%)$ while in patients having fibroid size less than $3 \mathrm{~cm}$ the most profound effect was seen in reduction in menorrhagia (45\%). It was observed that Mifepristone was more effective in reducing size of fibroid $(>40 \%)$, reduction in pain $(30 \%)$ and reducing menorrhagia $(50 \%)$ than Ulipristal Acetate in patients having fibroid size of less than $3 \mathrm{~cm}$.

This was a prospective double-blind case control study. 40 patients having uterine fibroids and symptoms related to it were enrolled in this study. Patients having any exclusion criteria were excluded from the study. These 40 patients were divided into 2 groups. Patients in Group A received using mifepristone $25 \mathrm{mg}$ and Group B patients received Ulipristal acetate $10 \mathrm{mg}$.

\section{Demographic data}

Out of the 40 studied cases Group A had a mean age of $38.6 \pm 5.8$ years and group B patients had a mean age of $39.4 \pm 6.1$ years. The mean age of the patients was comparable in both the groups and there was no statistically significant difference.

The analysis of presenting complaints of the patients revealed that the most common presenting complaint of the patients was pain during menstruation (24/40) followed by lower abdominal pain (14/40), excessive bleeding during mensuration (13/40) dyspareunia (12/40) and pain occurring between the cycles particularly at midcycle (4/40).

Table 1: Mean age of the cases in both the groups.

\begin{tabular}{|lll|}
\hline & Group A & Group B \\
\hline Mean Age & $38.6 \pm 5.8$ yeas & $39.4 \pm 6.1$ years \\
\hline
\end{tabular}

The diagnosis of uterine fibroid was confirmed by either transabdominal or transvaginal ultrasound depending upon the size of fibroid and marital status of the patients. Out of the studied case patients $60 \%$ patients had subserosal fibroids while $10 \%$ and $30 \%$ patients had submucosal and intramural fibroids respectively.

Out of 40 studied cases 20 patients had fibroid size less than $3 \mathrm{~cm}$ and remaining 20 patients had fibroid size in between 3 to $5 \mathrm{~cm}$. There was no patient having fibroid size of more than $5 \mathrm{~cm}$.

Table 2: Fibroid size in the studied cases.

\begin{tabular}{|llll|}
\hline Fibroid sive & $<3 \mathrm{~cm}$ & $3-5 \mathrm{~cm}$ & Total \\
\hline Group A & 10 & 10 & 20 \\
\hline Group B & 10 & 10 & 20 \\
\hline Total & 20 & 20 & 40 \\
\hline
\end{tabular}

All the patients either received mifepristone $25 \mathrm{mg}$ or Ulipristal acetate $10 \mathrm{mg}$ daily. The analysis of the effect of medical management by these drugs was done. The study found that Mifepristone had profound effect in reducing menorrhagia $(62 \%)$ pain reduction $(60 \%)$ in patients having fibroid size of $3-5 \mathrm{~cm}$. While in patients having fibroid size less than $3 \mathrm{~cm}$ the most profound effect was seen in reduction in menorrhagea $(50 \%)$. It was observed that mifepristone was less effective in pain reduction in patients having fibroid size less than $3 \mathrm{cms}$ $(30 \%)$.

Table 3: Reduction in symptoms in patients receiving Mifepristone (Group A).

\begin{tabular}{|c|c|c|c|}
\hline & $\begin{array}{l}\text { Reduction } \\
\text { in size }\end{array}$ & $\begin{array}{l}\text { Reduction } \\
\text { in pain }\end{array}$ & $\begin{array}{l}\text { Reduction in } \\
\text { menorrhagia }\end{array}$ \\
\hline $3-5 \mathrm{~cm}$ & $55 \%$ & $60 \%$ & $62 \%$ \\
\hline$<3 \mathrm{~cm}$ & $>40 \%$ & $30 \%$ & $50 \%$ \\
\hline
\end{tabular}

The analysis of group B patient receiving Ulipristal acetate revealed that it had predominant effect on blood loss and reduced blood loss was seen in $90 \%$ patients having fibroid size of 3-5 cm. Reduction in fibroid size and pain was significant in patients who received Ulipristal acetate and had fibroid of $3-5 \mathrm{~cm}$ while in patients having fibroid size less than $3 \mathrm{~cm}$ the Ulipristal acetate was found to be effective in terms of reduction in blood loss during menstrual periods (45\%). It was found 
to be less effective in terms of reduction in fibroid size and pain which was seen in $<20 \%$ and $22 \%$ respectively in patients having fibroid size of less $3 \mathrm{~cm}$.

Table 4: Reduction in symptoms in patients receiving Ulipristal acetate (Group B).

\begin{tabular}{|llll|} 
Reduction & $\begin{array}{l}\text { Reduction } \\
\text { in size }\end{array}$ & $\begin{array}{l}\text { Reduction in } \\
\text { menorrhagia }\end{array}$ \\
\hline $3-5 \mathrm{~cm}$ & $80 \%$ & $85 \%$ & $90 \%$ \\
\hline$<3 \mathrm{~cm}$ & $<20 \%$ & $22 \%$ & $45 \%$ \\
\hline
\end{tabular}

Thus, it was found that Mifepristone was more effective in patients having smaller fibroids (less than $3 \mathrm{~cm}$ ) as far as pain and size of the fibroid is concerned and Ulipristal acetate was more effective in medical management of the patients having fibroids of relatively larger size $(3-5 \mathrm{~cm})$. Ulipristal acetate was very effective in reducing the size and symptoms associated with fibroids in patients having size between 3 to $5 \mathrm{~cm}$.

\section{DISCUSSION}

Selective Progesterone receptor modulators (SPRMs) like mifepristone and Ulipristal Acetate have been used for the treatment of dysfunctional uterine bleeding and uterine myomas because of their antiproliferative effects on endometrium and myometrium. ${ }^{16}$ In 1993 Murphy et al first described use of mifepristone for the treatment of uterine fibroid. They showed uterine fibroids to be steroid hormone dependent tumors possessing Estrogen and progesterone receptors (ER and PR). ${ }^{17}$ They proposed that antiprogesterone reduce the size of uterine fibroids either by blocking the effect of progesterone or interference of estrogen action on fibroids. The authors examined the effects of daily administration of mifepristone $50 \mathrm{mg}$ for a period of 3 months in 10 patients with uterine fibroids. Baseline ultrasound examinations were obtained and repeated monthly during treatment as a measure of fibroid size. The authors found that fibroid volume $($ mean $\pm \mathrm{SE})$ decreased $21.9 \pm 4.8 \%$ after 4 weeks, $39.5 \pm 6.6 \%$ ( $\mathrm{P}<0.001)$ after 8 weeks, and $49.0 \pm 9.2 \%$ ( $\mathrm{P}<0.001)$ after 12 weeks of treatment compared to pre-treatment measurements. They further found that administration of mifepristone was associated with a significantly reduced immunoreactivity in fibroids as compared with tissues from untreated patients, this suggested that mifepristone caused regression of fibroids by through a direct antiprogesterone effect. The findings of the present study were similar, and we found that administration of mifepristone was associated in reduction in size of fibroids by $55 \%$ and $40 \%$ in patients having fibroid size of more than $3-5 \mathrm{~cm}$ and less than 3 $\mathrm{cm}$ respectively. The incidence of reduction in pain and menorrhagia was found to be $60 \%$ and $62 \%$ in patients with a fibroid size of $3-5 \mathrm{~cm}$ while it was $30 \%$ and $50 \%$ in patients having fibroid size of less than $3 \mathrm{~cm}$.

Feng $\mathrm{C}$ in their comparative study of women with symptomatic uterine fibroids who were treated with $5 \mathrm{mg}$ or $2.5 \mathrm{mg}$ of mifepristone or placebo found that treatment with mifepristone was associated with significant improvement in health-related quality of life. ${ }^{18}$

Similarly, the utility of Ulipristal acetate has been tested in many randomized controlled trials. The first large randomized controlled trial for use of Ulipristal acetate in medical management of fibroids (PEARL I) compared it with placebo in uterine fibroids. The study found that use of Ulipristal acetate was associated with significant reduction in menorrhagia and fond that bleeding was controlled in $91 \%, 92 \%$, and $19 \%$ of the women receiving UPA (5 $\mathrm{mg})$, UPA (10 $\mathrm{mg})$, and placebo, respectively. This staggering difference in control of bleeding made many researches take up the studies using Ulipristal acetate for medical management of uterine fibroids. PEARL II study was another randomized controlled trial comparing Ulipristal acetate with $\mathrm{GnRH}$ analog in the medical management of uterine fibroids. The study found that menorrhagia was controlled in $90 \%$, $98 \%$, and $89 \%$ of the women receiving Ulipristal acetate (5mg), Ulipristal acetate $(10 \mathrm{mg})$ and $\mathrm{GnRH}$ analogue, respectively. The authors concluded that Ulipristal acetate use was associated with a quicker control of menorrhagia as compared with GnRH analogues. The reduction of fibroid size, reduction in pain and decreased incidence of menorrhagia was associated with improved quality of life in patients receiving Ulipristal acetate. The findings were similar to the present study we found that administration of Ulipristal acetate $10 \mathrm{mg}$ daily was associated with reduction in fibroid size in $80 \%$ and $<20 \%$ in patients having fibroid size of $3-5 \mathrm{~cm}$ and less than $3 \mathrm{~cm}$ respectively. The incidence of reduction in pain and menorrhagia was found to be $85 \%$ and $90 \%$ in patients with a fibroid size of $3-5 \mathrm{~cm}$ while it was $22 \%$ and $45 \%$ in patients having fibroid size of less than 3 cm. ${ }^{19}$

Various other studies have shown the effectiveness of Ulipristal acetate in decreasing menorrhagia and improving the quality of life of patients with uterine fibroids. In a recent study Kalampokas $\mathrm{T}$ et al found that treatment of patients with Ulipristal acetate was associated with improved quality of life parameters and reduction in fibroid size. They concluded that Short-term use of Ulipristal acetate is effective and safe method of treating uterine fibroids. ${ }^{20}$ All these findings were similar to findings of our study which showed that administration of Ulipristal acetate was associated with decreased blood loss and fibroid size consequently causing improved quality of life.

\section{CONCLUSION}

Treatment of symptomatic fibroids by Mifepristone as well as Ulipristal acetate was associated with reduction in fibroid size, reduced blood loss and decreased pain. It was found that Ulipristal acetate was more effective when used in patients having fibroid size of $3-5 \mathrm{~cm}$. Mifepristone was found to be more effective when used 
in patients having fibroid size of less than $3 \mathrm{~cm}$. We conclude from this study that both these drugs can be used for treatment of symptomatic fibroids. Ulipristal acetate should be preferred over Mifepristone when the fibroid size is found to be more than $3 \mathrm{~cm}$.

Funding: No funding sources

Conflict of interest: None declared

Ethical approval: The study was approved by the Institutional Ethics Committee

\section{REFERENCES}

1. Khan AT, Shehmar M, Gupta JK. Uterine fibroids: current perspectives. Int J Women's Health. 2014;6:95-114.

2. Purohit P, Vigneswaran K. Fibroids and Infertility. Current Obstet Gynecol Reports. 2016;5:81-8.

3. Stewart EA, Laughlin-Tommaso SK, Catherino WH, Lalitkumar S, Gupta D, Vollenhoven B. Uterine fibroids. Nat Rev Dis Primers. 2016;2:16043.

4. Sparic R, Mirkovic L, Malvasi A, Tinelli A. Epidemiology of uterine myomas: a review. Int $\mathbf{J}$ Fertility Sterility. 2016;9(4):424-35.

5. Sue W, Sarah SB. Radiological appearances of uterine fibroids. Indian J Radiol Imaging. 2009;19(3):222-31.

6. Levens ED, Wesley R, Premkumar A, Blocker W, Nieman LK. Magnetic resonance imaging and transvaginal ultrasound for determining fibroid burden: implications for clinical research. Am J Obstet Gynecol. 2009;200(5):537.

7. Aluwee SAZBS, Kato H, Zhou X, Hara T, Fujita H, Kanematsu M, et al. Magnetic resonance imaging of uterine fibroids: a preliminary investigation into the usefulness of 3D-rendered images for surgical planning. Springer Plus. 2015;4:384.

8. Purslow CE. Fibroid uterus with red degeneration and with early gestation. Proceedings of the Royal Society of Medicine. Obstet Gynecol Sect. 1914;7:300.

9. Al Ansari AA, Al Hail FA, Abboud E. Malignant transformation of uterine leiomyoma. Qatar Med J. 2012;2012(2):71-4.

10. Divakar H. Asymptomatic uterine fibroids. Best Pract Res Clin Obstet Gynaecol. 2008;22(4):643-54.

11. Ciarmela P, Ciavattini A, Giannubilo SR, Lamanna $\mathrm{P}$, Fiorini R, Tranquilli AL, et al. Management of leiomyomas in perimenopausal women. Maturitas. 2014;78(3):168-73.

12. Han NL, Ong CL. Magnetic resonance-guided focused ultrasound surgery (MRgFUS) of uterine fibroids in Singapore. Ann Acad Med Singapore. 2014;43(11):550-8.

13. Song H, Lu D, Navaratnam K, Shi G. Aromatase inhibitors for uterine fibroids. Cochrane Database Syst Rev. 2013;(10):CD009505.

14. Murji A, Whitaker L, Chow TL, Sobel ML. Selective progesterone receptormodulators (SPRMs) for uterine fibroids. Cochrane Database Syst Rev. 2017;4:CD010770.

15. Arendas K, Leyland NA. Use of ulipristal acetate for the management of fibroid-related acute abnormal uterine bleeding. J Obstet Gynaecol Can. 2016;38(1):80-3.

16. Woźniak S, Szkodziak P, Czuczwar P. The effect of ulipristal acetate treatment on symptomatic uterine fibroids within 12-months follow-up. Przeglad Menopauzalny Menopause Rev. 2014;13(1):18-21.

17. Englund K, Blanck A, Gustavsson I, Lundkvist U, Sjöblom P, Norgren A. Sex steroid receptors in human myometrium and fibroids: changes during the menstrual cycle and gonadotropin-releasing hormone treatment. J Clin Endocrinol Metab. 1998;83(11):4092-6.

18. Feng C, Meldrum S, Fiscella K. Improved quality of life is partly explained by fewer symptoms after treatment of fibroids with mifepristone. International J Gynaecol Obstet. 2010;109(2):121-4.

19. Bourdet TA, Luton D, Koskas M. Clinical utility of ulipristal acetate for the treatment of uterine fibroids: current evidence. Int J Women's Health. 2015;7:321330 .

20. Kalampokas T, Kamath M, Boutas I. Ulipristal acetate for uterine fibroids: a systematic review and meta-analysis. Gynecol Endocrinol. 2016;32(2):91-6.

Cite this article as: Kale AR. Comparison between Mifepristone and Ulipristal acetate as an alternative to surgical management of uterine fibroids (leiomyoma) in symptomatic patients of reproductive age group in Asian population. Int J Reprod Contracept Obstet Gynecol 2018;7:109-13. 